\title{
Efficient control and Fault Detection and Isolation in Building HVAC systems

\author{
Avisek Naug
}

\author{
Vanderbilt University, Nashville, TN, USA, 37235 \\ avisek.naug@vanderbilt.edu
}

\begin{abstract}
My thesis work is focused on advancing the state of the art in smart building monitoring and control problems. A major part of my thesis will develop combined modeland data-driven models for the energy systems of buildings and apply them for energy monitoring and optimization, fault diagnosis, and fault-adaptive control. In past work, I have already developed initial machine learning-based models for energy optimization. In this paper, I am focusing on developing a framework using bond graphs to build a model of the HVAC system and understand how different factors affect the measurement variables of this system. This information will help us do subsequent fault diagnosis by studying the characteristics of the system behavior represented by the temporal causal graph. It will also provide the basis for fault-adaptive control of buildings.
\end{abstract}

\section{PROBLEM STATEMENT}

Large commercial buildings have elaborate HVAC systems that operate continuously to ensure comfortable conditions for their occupants. These buildings are monitored through a Building Automation System (BAS) which helps the building personnel to monitor whether the system is running under normal conditions. In the event of an abnormal measurement the system generates an alarm and alerts the personnel. The personnel then must manually look up which component has caused the alarm. Doing this process manually for a large distributed system can be a difficult.

To address this problem, I will describe, how I have developed a framework using the bond graphs to address the issue of fault detection and isolation in the large HVAC systems.

\section{CURRENT WORK: BOND GRAPHS FOR HVAC MODELING AND SUBSEQUENT FAULT DETECTION AND FAULT ISOLATION}

Bond Graph Model for the HVAC system: To perform any form of fault diagnosis we need to have a model of the system that can be used to simulate the behavior of

Avisek Naug. This is an open-access article distributed under the terms of the Creative Commons Attribution 3.0 United States License, which permits unrestricted use, distribution, and reproduction in any medium, provided the original author and source are credited. the same. This would be used to generate nominal data and deviation in measurements between simulated and actual system would help us in detecting faulty behavior. I modeled the HVAC system located at the Alumni Hall of Vanderbilt University (shown in Figure 2) using the bond graph approach.

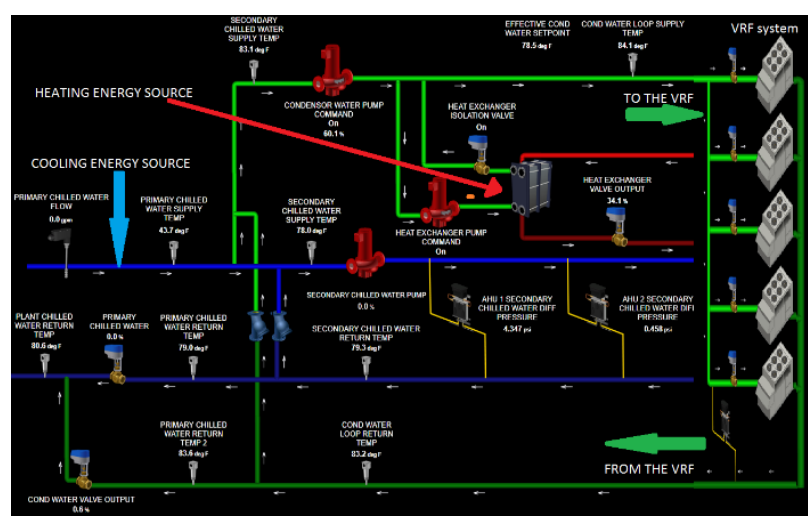

Figure 1: Chilled Water System at Alumni Hall

The three domains of interest in this work are the mechanical domain, hydraulic domain and the thermal domain description of the HVAC system. The mechanical domain includes the different pumps present in the pipeline which help build up pressure for the coolant, in this case the chilled water, to flow through different parts of the HVAC system. The transfer of power from mechanical effort to hydraulic effort is achieved through a modulated Gyrator whose conversion factor depends on the speed of rotation of the pump.

The hydraulic domain includes sources of flow which supply the chilled water to the HVAC system. The resistors are used to model the decrease of pressure through the length of the pipe due to pipe friction or change of elevation. There are several valves that modulate the flow of fluid in to different parts of the system and this is modeled by a Modulated Transformer in the bond graph.

The most important part of the HVAC model is the thermal domain bond graph as this is the main part that 
I am focusing on for my fault detection and isolation work. A convective resistor [2-3] is used to model the flow of heat energy through a fluid media for energy transfer. It is being placed throughout the thermal domain model because both the primary and the secondary chilled water lines are transferring fluid between different parts of the building using this water.

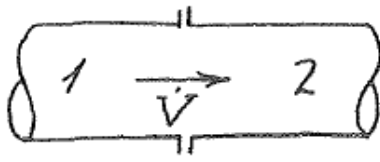

\section{Pipeline Port}

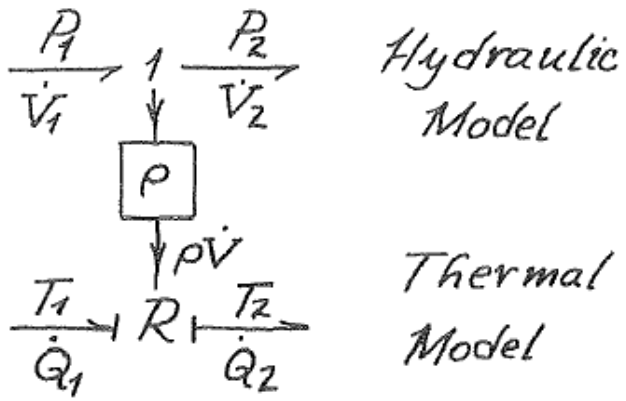

Figure 2: Convective Resistor

The transfer of energy between two sections shown in Figure 4 is dictated by the following two equations.

$$
\begin{array}{ll}
\dot{Q}_{1}=\dot{Q}_{2}=\rho c \dot{V} T_{1} ; & \text { if } \dot{V}>0 \\
\dot{Q}_{1}=\dot{Q}_{2}=\rho c \dot{V} T_{2} ; & \text { if } \dot{V}<0
\end{array}
$$

Where $\mathrm{Q}$ is the heat flow and $\mathrm{T}$ is the temperature at a source or the sink and $\dot{V}$ is the rate of flow of fluid with the appropriate sign. There are also normal resistive elements to model heat dissipation thorugh the material of the HVAC componenets and capacitive elements to model the heat capacity of the coils and pipe lines.

FDI using the temporal causal graphs: Using these models, I will derive the Temporal Causal Graphs for the HVAC system and use that graph for implicating different parameters using backward propagation and subsequent fault isolation using forward propagation.

The temporal causal graphs are used to establish a cause effect relation among power variables in the bond graph. They are derived from the bond graph using an extension of the SCAP algorithm [1]. The causal and integral relations from the bond graph are transformed to the TCG in the form of directed edges and integrating edges respectively and this helps encode causal information for tracing faults and propagating effects of parameter deviation on measured system variables.
One we have the TCG from the bond graph, the backward propagation method will help us propagate deviations, observed between measurements and nominal values from the simulated bond graph, backward along the edges of the temporal causal graph along with positive or negative labels on the upstream vertices/components to indicate whether an increase or a decrease in the component value would have caused such a deviation in the measured variable.

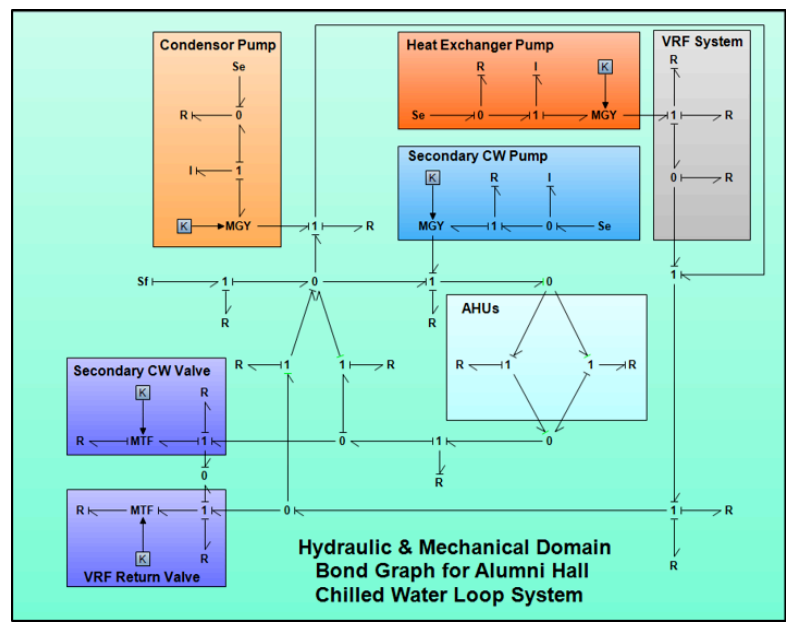

Figure 3: Hydraulic and mechanical domain bond graph of the HVAC system

Once we have a complete set of implicated parameters, we use the Temporal Causal Graph to see the predicted behavior of the model under individual parameter deviations. This method in FDI parlance would be termed forward propagation of the parameter deviations to the measured variables. While propagating parameter deviations forward, temporal edges in the Temporal Causal Graph would have an integrating effect on the subsequent variables. So the FDI framework would take in to account both zero and higher order effects.

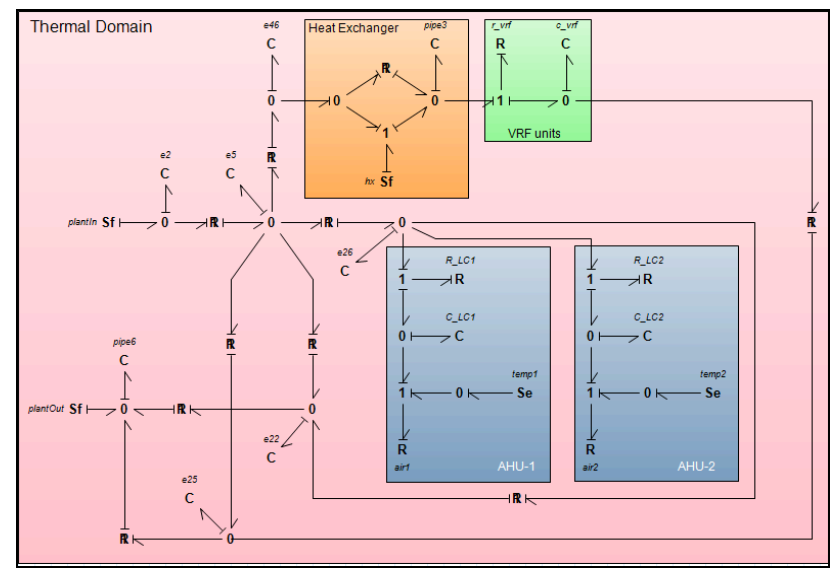




\section{Figure 4: Thermal Domain Bond Graph of the HVAC system}

\section{RESEARCH PLAN}

Using the Bond graphs for fault detection and isolation: This research focusses on applying the FDI framework mentioned in the previous section to other buildings as well and ultimately automate and integrate the process into the building Automation System for future work on fault adaptive control. As discussed there would be two parts to this:

- Developing a residual method based approach for fault detection

- Using forward and backward propagation method of TCG for fault isolation in the HVAC

Challenges: Developing models that faithfully replicates complex HVAC system behavior is the primary challenge in this problem. Accurate domain knowledge would be required for this problem.

\subsection{Work done on Machine learning models for energy consumption}

- I have developed a framework for learning the building energy consumption using the AdaBoost regression technique.

- Used Stochastic Gradient Descent to optimize the energy consumption using the set of control variables available in the system.

- Obtained on average $10 \%$ savings for each cluster.

\subsection{Work Performed on FDI using bond graphs}

- Developed the bond graph for the Mechanical and Hydraulic domain of the Chilled water system:

- This is shown in figure 3 where we have modeled the condenser pump, Heat Exchanger pump, Secondary Chilled Water pump.

- Modeled the general structure of the water flow with pressure drops across the pipe structure using the series resistors.

- The valves are denoted by a modulated transformer and the end resistors are the flow sink denoting water leaving the building.

- Developed the thermal domain bond graph of the HVAC system:

- This is shown in figure 4 where I have modeled the thermal domain of the bond graph. The convective resistors are used to carry the heat either away from the building or carry heat to the building depending on ambient temperature conditions.
- The heat exchanger is modeled as a source of flow which adds enthalpy to the chilled water circuit during extremely cold season.

- Developed a state space simulation model to validate that the model is correctly able to predict the measured/output variables so that it can be used for fault detection later on. The model can simulate the system behavior to a sufficient degree of accuracy as shown in Figure 5 below.

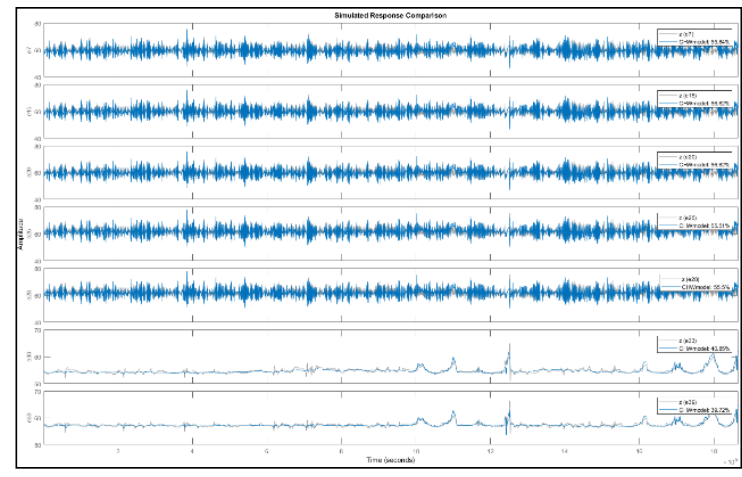

Figure 5: Comparison of simulated and actual response of different measured variables of the thermal domain bond graph in figure 4 .

\subsection{Remaining Work}

- Develop a Temporal Causal Graph from the bond graph structure.

- Use it to back propagate faults for hypothesizing the set of faults

- Use forward propagation for predicting future behavior under fault conditions and isolate the actual faulty component from the set of faults.

\section{CONCLUSION}

This work proposes a formal methodology for fault detection and isolation in HVAC systems. What makes the problem challenging is complexity of doing the FDI for the entire system since HVACs systems seem to be highly interconnected and a fault in one part of the system may arise due to abnormal behavior in a different part of the system.

\section{REFERENCES}

[1]Mosterman, P. J., \& Biswas, G. (1999). "Diagnosis of continuous valued systems in transient operating regions," in IEEE Transactions on Systems, Man, and Cybernetics - Part A: Systems and Humans, vol. 29, no. 6, pp. 554-565, Nov. 1999. doi: $10.1109 / 3468.798059$

[2] Karnopp D. Pseudo Bond Graphs for Thermal Energy Transport. ASME. J. Dyn. Sys. Meas., Control. 1978; 100(3):165-169. doi: $10.1115 / 1.3426363$.

[3] R. C. Rosenberg and D. Karnopp, Introduction to Physical Systems Dynamics. New York: McGraw-Hill, 1983. 